cannabis is not as harmless as we had thought earlier' - an amazing conclusion from a study where only $1 \%$ of the respondents identified as dependent reported social consequences of their use, while the most prevalent symptom $(10 \%)$ was persistent desire. In everyday parlance, they smoked because they liked it.

Use of the very broad categorisations of the DSM is especially worrisome. Clinicians using these guidelines apply them to people presenting with problems. The use of such categorisations in research, however, constitutes imprecise criteria to determine a person's dependence, resulting in the phenomenon being grossly overreported.

Researchers have been able to generate dependency by applying these same criteria to behaviours as diverse as jogging, shopping, sex, prayer and mountain climbing. In fact, these activities were found to be as addictive as cannabis (Franklin, 1990).

Problems include the disjunctive nature of the criteria (dependency can be ascribed to two people with absolutely no symptoms in common), and the essentially subjective way in which the characteristics are defined. The lack of specificity in the measurement of cannabis dependence results in subjective measures being presented as objective and an over-reliance on the interpretive framework brought to bear. How did the authors differentiate between 'wants' and what DSM characterises as 'needs'? Was this differentiation communicated to respondents? The study fails to differentiate respondents with no dysfunction associated with their dependence from those with significant cannabis-related problems.

Finally, the only index of consumption employed is frequency of use. This is most unsatisfactory; a 'smoke' is not a standardised measure and the consequent lack of any demonstrable association between tetrahydrocannabinol consumption and the dependence syndrome begs the question, dependent on what? Preparing a joint? Inhaling deeply?

Coffey, C., Carlin, J. B., Lynskey, M., et al (2003) Adolescent precursors of cannabis dependence: findings from the Victorian Adolescent Health Cohort Study. British Journal of Psychiatry, 182, 330-336.

Franklin, D. (1990) Hooked: not everyone become addicted. How come? Health, 4, 38.

Lawrence, J. (2003) Teenagers addicted to pot. Sunday Mail (Queensland), 6 April.

T. Palmer Youth Substance Abuse Service, Level I/I3I Johnstone Street, Fitzroy, Victoria 3065,

Australia. E-mail: tpalmer@ysas.org.au
Authors' reply: In response to Dr Miller we would like to state some general principles, to clarify our methodology and provide some additional results. First, we have no argument with the truism that causality cannot be inferred from correlation. Dr Miller seems to overlook the fact that, despite widespread awareness of the dangers of determining causality, the terms 'risk' and 'protective' are commonly used to describe associations identified in longitudinal studies. Indeed, identifying and interpreting such associations is the primary reason for conducting cohort studies. The reiteration of standard caveats should not be necessary in every article arising from these studies and would make for very tedious reading indeed.

The potential for inadequate control of confounding by unmeasured or omitted confounding factors is always a possibility in any multivariate analysis. Researchers are inevitably constrained by the measures they have at their disposal which, in turn, result from the constraints of research directions, design, responder burden and so on. Dr Miller criticises us for omitting socio-demographic measures while including correlated behavioural measures. In terms of the former, we assessed the influence of both parental education and metropolitan residence on cannabis dependence but as there was no evidence of univariate associations for either measure they were unlikely to be confounders (parental education, reference group 'some tertiary': completed secondary school OR 0.8 (95\% CI 0.5-1.3); incomplete secondary OR 1.0 (95\% CI 0.6-1.6); school in metropolitan Melbourne: OR 1.0 (95\% CI 0.6-1.5)). As they were uninformative, these findings were omitted from the article in the interests of parsimony and conserving space. As the report focused on adolescent behavioural and mental health predictors of cannabis dependence, both parental substance use and peer substance use, although likely to be predictors, were not considered relevant to the question. Indeed, they were omitted from the analysis as their inclusion could have masked the associations of interest, exactly as Dr Miller describes.

We acknowledge that confounding occurred between some of the explanatory measures included in the multivariate analysis. We illustrated and discussed in some detail the confounding that occurred between early-onset cannabis use, cigarette smoking and antisocial behaviour.
Furthermore, the interaction between problematic alcohol use and weekly cannabis use to which Dr Miller objects arose as post hoc examination of confounding.

Mr Palmer misunderstands the denominator of the reported symptom prevalences: we described overall symptom prevalence in the 1601 participants. Symptom prevalences in participants classified as being cannabis dependent were reported in an earlier publication and were: tolerance $17 \%$, withdrawal $74 \%$, unintentioned use $84 \%$, persistent desire $91 \%$, excessive time spent obtaining, using or recovering from use $74 \%$, social consequences of use $18 \%$ and continued use despite acknowledged health problems $63 \%$ (Coffey et al, 2002). Furthermore, participants classified as dependent cannabis users reported compulsive and out-of-control use more frequently than those classified with dependent alcohol use. That there is gathering evidence of social, physical and mental health harm, including dependence, arising from longterm cannabis use is now beyond debate. For a brief and informative review of the current literature on this topic see Ashton (2002).

Mr Palmer debates what really constitutes cannabis dependence. That young people 'are smoking because they like it' does not preclude the possibility that they may be dependent. Alternatively, they may be using it to stop feeling awful, in the self-medication paradigm. He quotes an assertion that other non-challenging behaviours performed persistently may also fit dependence criteria. This may be so, but the harm that arises from these activities is a moot point. The issue that concerns us, and that we used the current gold standard instrument in population research to identify, is that cannabis dependence inevitably prolongs heavy use. No measure applied at interview can be considered to be completely sensitive and specific for all the reasons that Mr Palmer states but the unreferenced assertion that the 'phenomena [are] grossly overreported' is unsupportable in the light of extensive developmental and confirmatory work performed in treatment and non-treatment settings (e.g. Nelson et al, 1999). We do not consider it a problem that individuals can be classified as dependent with different combinations of symptoms conversely, we need to increase our understanding of symptom combinations and their significance (Nelson et al, 1999). 
The final point that Mr Palmer makes is to query the validity of our measure of cannabis use. He appears to have misread the definition - we did not ask about 'smokes' at all. We asked participants how often they 'used cannabis' without specifying the method of delivery. We assume the word 'used' is unambiguous and involves ingestion in some manner.

Finally, we follow no political agenda but seek only to inform the general public and policy makers using sound epidemiological evidence resulting from good study design, careful analysis and cautious interpretation. Our article represents a step towards filling the evidence void in the current polarised debate about important public health and policy issues surrounding cannabis use (Strang et al, 2000).

Ashton, H. (2002) Cannabis or health? Current Opinion in Psychiatry, 15, 247-253.

Coffey, C., Carlin, J. B., Degenhardt, L., et al (2002) Cannabis dependence in young adults: an Australian population study. Addiction, 97, 187-194.

Nelson, C. B., Rehm, J., Uston, T. B., et al (1999) Factor structures for DSM-IV substance disorder criteria endorsed by alcohol, cannabis, cocaine and opiate users: results from the $\mathrm{WHO}$ reliability and validity study. Addiction, 94, 843-855.

Strang, J., Witton, J. \& Hall, W. (2000) Improving the quality of the cannabis debate: defining the different domains. BMJ, 320, 108-110.

C. Coffey Centre for Adolescent Health, 2 Gatehouse Street, Parkville 3052, Victoria, Australia. E-mail: coffey@cryptic.rch.unimelb.edu.au

J. B. Carlin Murdoch Children's Research Institute and University of Melbourne, Australia M. Lynskey Washington University, St Louis, Missouri, USA

G. C. Patton Murdoch Children's Research Institute, Parkville, Victoria, Australia

\section{MRCPsych exams}

I read with interest the informative editorial on the MRCPsych examination by Dr Tyrer and Professor Oyebode (2004). I agree with the authors' view that examinations require continuous assessment and refinement and also note their admission that political and external factors are likely to drive further changes.

However, I am still puzzled to note their ambiguity over defining the direction of change in the future. They give three examples of potential future directions: modularisation of courses with assessment at the conclusion of modules; continuation of high-stakes tests; and regrading of the record of in-service training (RITA) as an exit examination at the completion of higher specialist training. However, their description of these examples is vague.

This is an era of heightened societal expectations, increased regulatory control and external scrutiny of professionals. There remains at least a theoretical possibility of external quality assurance standards and mechanisms being imposed on the medical Royal Colleges, including the Royal College of Psychiatrists.

Eraut (1994) has argued that a professional's competence has at least two dimensions, scope and quality. Scope concerns what a person is competent in - the range of roles, tasks and situations for which their competence is established or may be reliably inferred. Quality concerns judgements on the quality of that work along a continuum. Determining the acceptable and measurable cut-off points on the quality dimension for senior house officers, specialist registrars and consultants remains an important task for the profession.

Schön (1987) has argued that if professions are blamed for ineffectiveness and impropriety, their schools are blamed for failing to teach the rudiments of effective and ethical practice. Greater emphasis on the processes of training, reflective practice, training the trainers, continuing professional development, relevant educational research and interprofessional learning would help to sustain and enhance the profile of psychiatry in the society. The profession requires a clear direction from its leaders.

Eraut, M. (1994) Concepts of competence and their limitations. In Developing Professional Knowledge and Competence, pp. 163-181. London: Falmer.

Schön, D. A. (1987) Preparing professionals for the demands of practice. In Educating the Reflective Practitioner, pp. I-2I. San Francisco, CA; London: JosseyBass.

Tyrer, S. \& Oyebode, F. (2004) Why does the MRCPsych examination need to change? British journal of Psychiatry, 184, 197-199.

R. A. Faruqui Charing Cross Scheme \& Imperial College London, Charing Cross Hospital, Fulham Palace Road, London W6 8RF, UK
Authors' reply: We have noted Dr Faruqui's comments on our editorial. Dr Faruqui believes we should be more specific about recommendations for psychiatry examinations in the future, and argues that we have been ambiguous in not defining the format for future psychiatry examinations in more detail.

The Royal College of Psychiatrists is not able independently to direct the course of examinations in the future. The Postgraduate Medical Educational and Training Board has indicated what principles should hold in postgraduate examinations, and the Royal College of Psychiatrists follows these as well as observing the practices of the other medical Royal Colleges.

There is a move to include workplace assessments as part of the panoply of assessment of competence. The methods to achieve this have not yet been fully described or, indeed, evaluated. The degree to which this kind of assessment will form part of the assessment of a candidate in a future MRCPsych examination has not been made explicit.

This is the present state of affairs. We are not expressing our own opinions in this part of the editorial; we are indicating the present state of play. We believe that competence is judged by public examinations and that performance is measured by workplace assessments that approximate to what occurs in the real world. Quality of work is not assessed in examinations and we do not believe that this is part of the remit of examination boards.

\section{Declaration of interest}

S.T. is the immediate past Chief Examiner of the Royal College of Psychiatrists and F.O. is the present Chief Examiner and is a member and examiner of the Professional Licensing Assessment Board of the General Medical Council.

S. Tyrer University of Newcastle uponTyne, Royal Victoria Infirmary, Newcastle uponTyne NEI 4LP,UK

F. Oyebode South Birmingham Mental Health NHS Trust, Queen Elizabeth Psychiatric Hospital, Mindelsohn Way, Edgbaston, Birmingham BI5 2QZ, UK 